\title{
Adaptive Formation Tracking Control of Directed Networked Vehicles in a Time-Varying Flowfield
}

\author{
Yang-Yang Chen* \\ Southeast University, Nanjing, 210096, China, \\ Kaiwen Chen $^{\dagger}$ \\ Imperial College London, London, SW7 2AZ, U.K. \\ Alessandro Astolfi $\ddagger$ \\ Imperial College London, London, SW7 2AZ, U.K. \\ University of Rome, Tor Vergata, 00133 Rome, Italy
}

\section{Nomenclature}

$$
\begin{array}{ll}
\mathbb{R} & =\text { the set of real numbers } \\
\mathbb{R}^{n \times m} & =\text { the set of } n \times m \text { matrices with real entries } \\
I & =\text { the identity matrix } \\
0 & =\text { the zero matrix of appropriate dimension } \\
\mathbb{T} & =\text { the one-torus } \\
\|\cdot\| & =\text { the Euclidean norm } \\
|M| & =\text { the determinant of matrix } M \\
\otimes & =\text { the Kronecker product } \\
p_{i} & =\text { the position of vehicle } i \\
p_{x_{i}} & =\text { the position abscissa of vehicle } i \\
p_{y_{i}} & =\text { the position ordinate of vehicle } i \\
u_{i} & =\text { the control input of vehicle } i \\
u_{x_{i}} & =\text { the control input of vehicle } i \text { along the abscissa } \\
u_{y_{i}} & =\text { the control input of vehicle } i \text { along the ordinate } \\
f_{i} & =\text { the flow velocity at position } p_{i} \text { and time } t \\
\lambda_{i} & =\text { the path following error of vehicle } i \\
\Omega_{i} & =\text { an open set associated with vehicle } i \\
f_{\alpha_{i}} & =\text { the flow amplitude at } \lambda_{i} \\
f_{\beta_{i}} & =\text { the basis flow function evaluated at position } p_{i} \text { and time } t
\end{array}
$$

\footnotetext{
*Associate Professor, School of Automation and Key Laboratory of Measurement and Control of Complex Systems of Engineering, Ministry of Education, yychen@seu.edu.cn.

${ }^{\dagger} \mathrm{PhD}$ Student, Department of Electrical and Electronic Engineering, kaiwen.chen16@imperial.ac.uk.

‡Professor, Department of Electrical and Electronic Engineering and Department of Civil Engineering and Computer Science Engineering, a.astolfi@imperial.ac.uk
} 


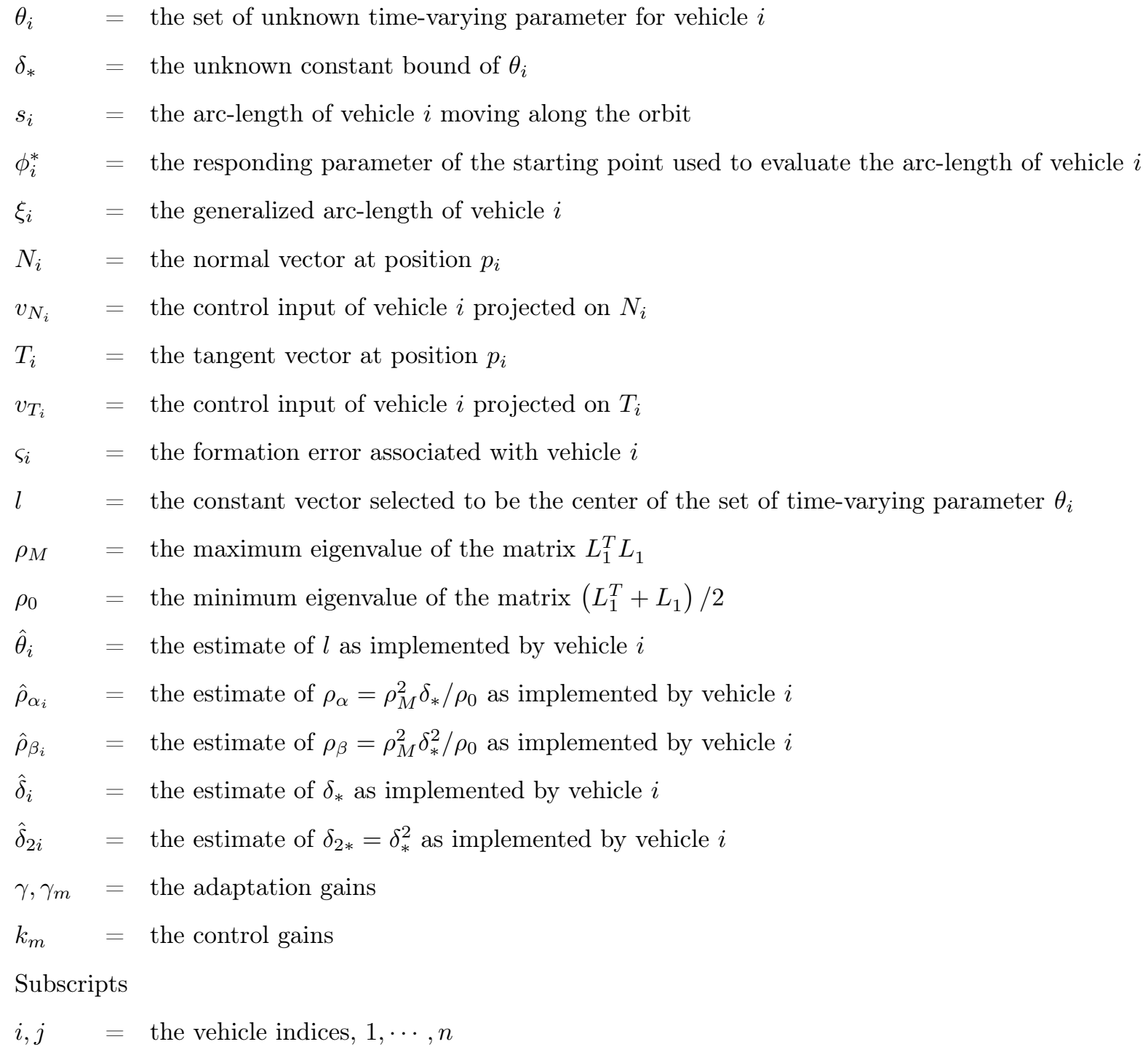

\section{Introduction}

$\mathrm{T}^{\mathrm{N}}$ the past decade there has been growing interest in the use of formation tracking control technology for 1 oceanic and planetary explorations [1]3]. Formation tracking control, which requires multiple vehicles to simultaneous follow a set of given orbits with a desired formation, can achieve better performance than a single monolithic vehicle in seeking measurements of biological variables across a range of spatial and temporal scales. Typical approaches in this line of research deal with each vehicle's motion without the effect of external flowfields; see for example in [4 -7. However, the motion of each vehicle is inevitably affected by external flowfields. More importantly, the flowfields may force the vehicles to deviate from the desired orbit and even disrupt the formation [8], which yields the urgency of designing formation tracking methods in the presence of flowfields.

Many studies of flow-based formation tracking control exploit bidirectional communication topologies. In 
9], two proposed circular formation control laws in the presence of a time-invariant flowfield were presented in the case of all-to-all topology and undirected, connected, topology, respectively. In [10], the circular formation control methods introduced in [9] have been modified by using a state observer, which permits an unknown time-invariant flowfield and a flowfield uniformly rotating at an unknown constant angular velocity. In [11, formation tracking is achieved in the presence of a parameterized flowfield with unknown constant parameters by using a consensus-based filter. The formation tracking control laws are designed based on an adaptive neural network (ANN) in [12] and on an adaptive method in [13]. In addition to the aforementioned methods, consensus in the presence of disturbances has been investigated and can help in the solution of the flow-based formation tracking control problem. In [14], a consensus problem is solved with an adaptive estimation algorithm in the case of jointly connected topologies. In [15, a disturbance observer solves the leader-following consensus problem, under the assumption that the time derivative of the disturbance converges to zero and the communication topology of the followers is bidirectional. Finally, [16] presents an alternative filter for the consensus problem, assuming the unknown nonlinearities satisfy a Lipschiz-like condition.

In some complex environments, especially underwater, considering a directed communication topology is more realistic [17]. In the cases of sensor networks (e.g., navigation beacons [18]) some sensors/agents may have transceivers, while others may only have receivers. Even if bidirectional communication is achievable, such a method is not preferable because of the limited compatibility and power requirement of the communication equipment. It is therefore essential to design formation tracking methods for directed communication topologies, a problem which has not yet been extensively studied.

An adaptive formation tracking control problem with unknown constant parameters is considered in the case of directed and ring topologies in [19] and in the case of strongly connected topologies in [20]. In [21], ANNs are exploited for formation tracking in the case of a spanning tree, yet using ANNs only guarantees uniform boundedness of the tracking error. Moreover, observer-based cooperative output regulation algorithms for exosystem-like disturbances are designed for leader-following digraphs in 22 and for time-varying digraphs in [23]. The dynamics of the flowfield are often spatio-temporal and contain unknown time-varying parameters [24. The aforementioned methods cannot be used for solving the formation tracking control problem of vehicles in the case of a directed communication topology, especially in a spatio-temporal flowfield without restrictions on the rate of parameter variations. To cope with unknown time-varying parameters, a novel adaptive method, the congelation of variables method, has been proposed in 25] 28. This method has been exploited to solve the formation tracking control problem in the presence of an unknown spatio-temporal flowfield and bidirectional topologies in [29].

This paper intends to solve the robust formation tracking control problem for vehicles with first order dynamics connected by a directed topology. The flowfield under consideration is a spatio-temporal flow with 
unknown time-varying parameters; the only restriction is that the parameters are bounded. Different from our previous result for bidirectional topologies [29], in this paper, the topology associated to the virtual leader and the followers is directed. This topology is similar to what discussed in [1, for deep-space formation flying of leader and follower spacecrafts, in [3, for AUVs adaptive sampling in Monterey Bay, and in 4], for spacecraft formation flying based on a virtual structure. By integrating estimation algorithms for the eigenvalues of the Laplacian matrix and an unknown bound on the time-varying parameters into the congelation of variables method, a novel distributed formation tracking control law to achieve asymptotic converge with a stationary virtual leader is designed. A novel formation adjustment unit for the virtual leader provides formation movement with a desired speed.

The paper has the following outline. Section II provides some preliminaries and formulates the adaptive formation tracking control problem in the presence of an unknown spatio-temporal flowfield. Section III gives a solution for the problem for the case in which the virtual leader is stationary and in the case in which the virtual leader moves along an orbit. Simulation results are presented in Section IV. Conclusions are given in Section V.

\section{Problem Formulation}

Consider a formation composed of a virtual leader, labeled as the 0th vehicle, and $n$ followers, labeled as the 1 st to the $n$th vehicles. Each follower is position in a fixed inertial reference frame evolves according to the dynamic equation

$$
\dot{p}_{i}=v_{i}+f_{i}\left(p_{i}, t\right)
$$

where $p_{i}(t)=\left[p_{x_{i}}(t), p_{y_{i}}(t)\right]^{T} \in \mathbb{R}^{2}$ denotes the position, $v_{i}(t)=\left[v_{x_{i}}(t), v_{y_{i}}(t)\right]^{T} \in \mathbb{R}^{2}$ denotes the control input, and $f_{i}: \mathbb{R}^{2} \times \mathbb{R} \rightarrow \mathbb{R}^{2}$ denotes the action of the spatio-temporal flowfield. The dynamics of the virtual leader, that is the 0 th vehicle, are

$$
\dot{p}_{0}=v_{0},
$$

where $p_{0}(t)=\left[p_{x_{0}}(t), p_{y_{0}}(t)\right]^{T} \in \mathbb{R}^{2}$ and $v_{0}(t)=\left[v_{x_{0}}(t), v_{y_{0}}(t)\right]^{T} \in \mathbb{R}^{2}$ are its position and control input, respectively.

The network topology of the formation can be described by a digraph $\mathcal{G}=\{\mathcal{V}, \mathcal{E}\}$ in which the vertices/nodes $\mathcal{V}=\left\{\mathcal{V}_{0}, \cdots, \mathcal{V}_{n}\right\}$ are associated to the virtual leader and the $n$ vehicles, respectively, and $\mathcal{E} \subseteq \mathcal{V} \times \mathcal{V}$ is a set of network links. A directed path from node $\mathcal{V}_{j}$ to node $\mathcal{V}_{i}$ is a sequence of edges $\left(\mathcal{V}_{i}, \mathcal{V}_{i_{1}}\right)$, $\left(\mathcal{V}_{i_{1}}, \mathcal{V}_{i_{2}}\right), \ldots,\left(\mathcal{V}_{i_{l-1}}, \mathcal{V}_{i_{l}}\right),\left(\mathcal{V}_{i_{l}}, \mathcal{V}_{j}\right)$ in the network topology with distinct nodes $\mathcal{V}_{i_{k}}, k=0, \ldots, m$. A digraph is called a directed tree if there exists a node, called the root, that has directed paths to all other nodes of 
the digraph. Let, for $i, j=0, \ldots, n, a_{i j}=1$ if $\left(\mathcal{V}_{j}, \mathcal{V}_{i}\right) \in \mathcal{E}$ and $a_{i j}=0$ otherwise. In addition, define the Laplacian matrix $L \triangleq\left[l_{i j}\right]_{i, j=0}^{n}$ with $l_{i i}=\sum_{j=1}^{n} a_{i j}$ and $l_{i j}=-a_{i j}$, for any $i \neq j, i, j=0, \ldots, n$. For the considered formation the Laplacian matrix $L$ can be written as

$$
L=\left[\begin{array}{ll}
l_{00} & \beta_{0} \\
\alpha_{0} & L_{1}
\end{array}\right],
$$

where $\alpha_{0}=\left[l_{10}, \cdots, l_{n 0}\right]^{T}, \beta_{0}=\left[l_{01}, \cdots, l_{0 n}\right]^{T}$, and $L_{1} \in \mathbb{R}^{n \times n}$. If there is no path from the followers to the leader, then $l_{00}=0$ and $\beta_{0}=0$. This observation motivates the following assumption.

Assumption 1. The digraph consisting of the virtual leader and the $n$ vehicles contains a directed spanning tree with root $\mathcal{V}_{0}$.

Lemma 1 [30]. Consider the digraph $\mathcal{G}$. Suppose that Assumption 1 holds. Then $L_{1}$ is a nonsingular $M$-matrix and all eigenvalues of $L_{1}$ have positive real parts.

The formation tracking task consists of a path-following task and a formation-motion task. In the former, the desired orbit associated to each vehicle is a simple, closed, and regular curve with nonzero curvature. As discussed in detail in [7] the path-following error associated with the $i$ th vehicle can be defined by a smooth function $\lambda_{i}: \Omega_{i} \rightarrow\left(-\varepsilon_{i}, \varepsilon_{i}\right)$, where $\Omega_{i} \subset \mathbb{R}^{2}$ is an open set and $\varepsilon_{i}$ is a positive constant. As shown in Figure 1a, the path-following task is achieved if, along the trajectories of the system,

$$
\lim _{t \rightarrow \infty} \lambda_{i}\left(p_{i}(t)\right)=0
$$

and $p_{i}(t) \in \Omega_{i}$, for all $t \geq 0$, where

$$
\Omega_{i}=\left\{p_{i} \in \mathbb{R}^{2}|| \lambda_{i}\left(p_{i}(t)\right) \mid<\varepsilon_{i}\right\}
$$

Note that the formation preserves the relative positions along the paths and the arc-length can be regarded as the positions on the path. It is natural to use generalized arc-lengths to define the formation [6, 7, 13, 20, 29]. The arc-length is

$$
s_{i}\left(\lambda_{i}, \phi_{i}\right) \triangleq \int_{\phi_{i}^{*}}^{\phi_{i}} \frac{\partial s_{i}\left(\lambda_{i}, \tau\right)}{\partial \tau} d \tau
$$

where $\phi_{i}^{*}$ is the parameter associated with the starting point of the arc. Then, consistent with [7, the generalized arc-lengths $\xi_{i}\left(s_{i}\right)$ are $C^{1}$ functions of $s_{i}$ and $\left|\partial \xi_{i} / \partial s_{i}\right| \geq \varepsilon>0$. The generalized arc-lengths are selected in such a way that if the formation is achieved then

$$
\lim _{t \rightarrow \infty}\left(\xi_{i}(t)-\xi_{0}(t)\right)=0
$$




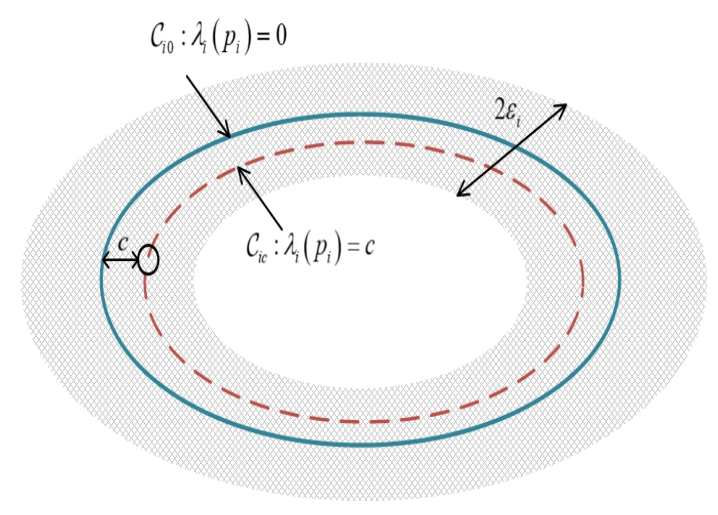

(a)

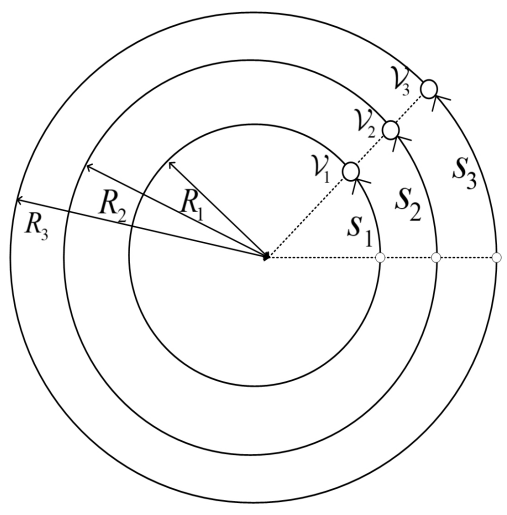

(b)

Fig. 1 Sketches of path followed and of formation. The meshed area denotes the set $\Omega_{i}$

Similarly to [29], assume that the flowfield is a unknown spatio-temporal flow parameterized as

$$
f_{i}\left(p_{i}, t\right)=f_{\alpha_{i}}\left(\lambda_{i}\right) f_{\beta_{i}}\left(p_{i}, t\right) \theta_{i}(t)
$$

where $f_{\alpha_{i}}: \mathbb{R} \rightarrow \mathbb{R}$ denotes the known amplitude of the flowfield; $f_{\beta_{i}}: \mathbb{R}^{2} \times \mathbb{R} \rightarrow \mathbb{R}^{2 \times m}$ is the $2 \times m$ matrix of known bounded basis functions of the flowfield; and $\theta_{i}(t) \in \mathbb{R}^{m}$ is the set of unknown time-varying parameters. Eq.17) can model, for example, the time-varying spatial flowfield in [10], the parameterizable flowfield in [11] and the Eulerian flowfield in [13.

Remark 1. The $C^{1}$ invertible mappings $\xi_{i}: \mathbb{R} \rightarrow \mathbb{R}$ define a change of coordinates that allows formulating the formation tracking problem with state variables $s_{i}$ into the consensus problem described by Eq. (6), with state variables $\xi_{i}\left(s_{i}\right)$. To form the desired formation, the desired arc-length $s_{i}^{*}$ of the $i$ th follower vehicle is determined by the arc-length of the leader $s_{0}$ via $s_{i}^{*}=g_{s_{i} s_{0}}\left(s_{0}\right)$, where $g_{s_{i} s_{0}}: \mathbb{R} \rightarrow \mathbb{R}$ is an invertible mapping explicitly defined by the desired formation. By Eq. [6], $\xi_{0}$ and $\xi_{i}$ are such that

$$
\xi_{i}\left(s_{i}^{*}\right)=\xi_{0}\left(s_{0}\right)
$$

Noting $s_{0}=g_{s_{i} s_{0}}^{-1}\left(s_{i}^{*}\right)$ yields $\xi_{i}=\xi_{0} \circ g_{s_{i} s_{0}}^{-1}$, which properly defines $\xi_{i}$ for any given $\xi_{0}$, since $g_{s_{i} s_{0}}$ is known. To clarify, consider the tracking problem depicted by Figure 1b, in which the vehicles move along concentric circles with radii $R_{i}$ and the desired formation is the radially in-line formation; that is, all vehicles stay on the same radius when moving along the circles. A natural selection of $\xi_{0}$ is the azimuth associated with $s_{0}$, namely $\xi_{0}\left(s_{0}\right)=\frac{1}{R_{0}} s_{0}$; note that being in the desired formation implies $g_{s_{i} s_{0}}\left(s_{0}\right)=\frac{R_{i}}{R_{0}} s_{0}$. The corresponding 
$\xi_{i}$ is given by

$$
\xi_{i}\left(s_{i}\right)=\xi_{0} \circ g_{s_{i} s_{0}}^{-1} \circ s_{i}=\frac{1}{R_{i}} s_{i}
$$

Assumption 2 [29]. The functions $f_{\alpha_{i}}$ are bounded and satisfy $\nabla \Psi_{i} f_{\alpha_{i}} \geq f_{\alpha_{i}}^{2}$, for all $\lambda_{i} \in\left(-\varepsilon_{i}, \varepsilon_{i}\right)$, and $f_{\alpha_{i}}(0)=0$, where $\nabla \Psi_{i}$ is the derivative of the barrier function $\Psi_{i}:\left(-\varepsilon_{i}, \varepsilon_{i}\right) \rightarrow \mathbb{R}$ with barrier $2 \varepsilon_{i}$.

Assumption 3. There exists a constant $c_{1}>0$ such that $\left\|f_{\beta_{i}}\right\| \leq c_{1}$, for all $p_{i} \in \Omega_{i}$ and $t \geq 0$.

Assumption 4 [28]. The vectors of unknown time-varying parameters $\theta_{i}$ is piecewise continuous and $\theta_{i}(t) \in \Theta_{i}$, for all $t \geq 0$, where $\Theta_{i}$ is a compact set possibly unknown. Moreover the radius of $\Theta_{i}$ satisfies

$$
\left|\theta_{i}(t)-l_{i}\right| \leq \delta_{*}
$$

where a constant vector $l_{i} \in \mathbb{R}^{m}$ is selected to be the center of the parameter set $\theta_{i}$ and $\delta_{*}$ is a unknown constant.

Remark 2. The open interval $\left(-\varepsilon_{i}, \varepsilon_{i}\right)$ is the range of the smooth function $\lambda_{i}$. The orbit associated to $\mathcal{C}_{i 0}$ is such that $\lambda_{i}\left(p_{i}\right)=0$, which implies that the positions of the agents are on the given orbits. We do not require the condition $\lambda_{i}\left(p_{i}(0)\right)=0$.

Remark 3. The path-following task dictates that each vehicle satisfies Eq. (3). The formation task dictates that each vehicle follows the virtual leader and maintains the desired relative positions with its neighbors along given orbits, that is, Eq. (6) holds. When both tasks are achieved, the agents move along the closed orbit at the desired speed and their trajectories can be naturally described by periodic solutions in the Euclidean space.

Remark 4. Eq. (7) provides a linearly parameterizable expression of a time-varying flowfield such that the effect of the flowfield disappears on the desired orbit associated to each vehicle. For example, consider a rotating flowfield

$$
f_{i}(t)=\tanh \left(1-\sqrt{p_{x_{i}}^{2}+p_{y_{i}}^{2}}\right) e^{j \sigma(t)} \vartheta_{i}
$$

where $j$ is the imaginary unit, $\vartheta_{i} \in \mathbb{R}$ is the magnitude of the flow, and $\sigma(t) \in \mathbb{R}$ is the rotation rate. Due to the fact that $\lambda_{i}\left(p_{i}\right)=1-\sqrt{p_{x_{i}}^{2}+p_{y_{i}}^{2}}$ when the given orbit is a unit circle, we have that $\tanh \left(1-\sqrt{p_{x_{i}}^{2}+p_{y_{i}}^{2}}\right)=$ $\tanh \lambda_{i}$. Let $f_{\alpha_{i}}=\tanh \lambda_{i}, f_{\beta_{i}}=I$, that is the the $2 \times 2$ identity matrix, and $\theta_{i}=e^{j \sigma(t)} \vartheta_{i}$. The above rotating flowfield can be rewritten as in Eq. (7).

Remark 5. Assumption 2 requires that the signs of $f_{\alpha_{i}}$ and $\nabla \Psi_{i}$ are consistent and $\left|f_{\alpha_{I}}\right| \leq\left|\nabla \Psi_{i}\right|$. The condition $f_{\alpha_{i}}(0)=0$ means that the effect of the flowfield disappears on the desired orbit associated to each vehicle. 
As a result the corresponding problem studied in the paper can be formulated as follows.

Formation tracking problem with a virtual leader. Let $i \in[1, n]$. Consider the system (1) and the initial position $p_{i}(0) \in \Omega_{i}$. Suppose Assumptions 1 to 4 hold. Design a formation tracking control $u_{i}$, with an adaptive estimate for the unknown time-varying flow parameter $\theta_{i}$, such that the closed-loop system satisfies the conditions (3), (4) and (6).

\section{Main Results}

\section{A. Open-Loop System}

Let $\theta(t)=\left[\theta_{1}^{T}(t), \cdots, \theta_{n}^{T}(t)\right]^{T} \in \mathbb{R}^{n m}$ and $\bar{f}_{\beta_{i}}\left(p_{i}, t\right)=\left[0_{2 \times m(i-1)}, f_{\beta_{i}}\left(p_{i}, t\right), 0_{2 \times m(n-i)}\right] \in \mathbb{R}^{2 \times n m}$ and rewrite $(7)$ as ${ }^{*}$

$$
f_{i}\left(p_{i}, t, \theta_{i}(t)\right)=f_{\alpha_{i}}\left(\lambda_{i}\right) \bar{f}_{\beta_{i}}\left(p_{i}, t\right) \theta(t) .
$$

To obtain the path-following dynamics for the $i$ th follower it is sufficient to differentiate $\lambda_{i}$, yielding

$$
\dot{\lambda}_{i}=\left\|\nabla \lambda_{i}\right\| v_{N_{i}}+g_{\lambda_{i}} f_{\beta_{N i}} \theta
$$

where $v_{N_{i}}=N_{i}^{T} v_{i}$ denotes the control input projected on the vector $N_{i}$ normal to the level orbit of the current position of the vehicle, that is, $N_{i}=\frac{\nabla \lambda_{i}}{\left\|\nabla \lambda_{i}\right\|}, f_{\beta_{N i}}=N_{i}^{T} \bar{f}_{\beta_{i}}$, and $g_{\lambda_{i}}=\left\|\nabla \lambda_{i}\right\| f_{a_{i}}$. For the $i$ th follower, differentiating both sides of Eq.(5) yields

$$
\dot{s}_{i}=v_{T_{i}}+\frac{\partial s_{i}}{\partial \lambda_{i}}\left\|\nabla \lambda_{i}\right\| v_{N_{i}}+f_{\alpha_{i}} f_{\theta_{i}} \theta
$$

where $v_{T_{i}}=T_{i}^{T} v_{i}$ denotes the control input projected on the vector $T_{i}$ tangent to the level orbit of the current position of the vehicle, that is, $T_{i}=\left[R_{1}, R_{2}\right]^{T} N_{i}$, with $R_{1}=[0,1]^{T}$ and $R_{2}=[-1,0]^{T}$. Note that $f_{\theta_{i}}=f_{\beta_{T i}}+\frac{\partial s_{i}}{\partial \lambda_{i}}\left\|\nabla \lambda_{i}\right\| f_{\beta_{N i}}$ and $f_{\beta_{T i}}=T_{i}^{T} \bar{f}_{\beta_{i}}$. As a result, the dynamics of $\xi_{i}$ are

$$
\dot{\xi}_{i}=\frac{\partial \xi_{i}}{\partial s_{i}} v_{T_{i}}+g_{\xi_{\alpha i}}+g_{\xi_{\beta i}} f_{\theta_{i}} \theta
$$

where $g_{\xi_{\alpha i}}=\frac{\partial \xi_{i}}{\partial s_{i}} \frac{\partial s_{i}}{\partial \lambda_{i}}\left\|\nabla \lambda_{i}\right\| v_{N_{i}}$ and $g_{\xi_{\beta i}}=\frac{\partial \xi_{i}}{\partial s_{i}} f_{\alpha_{i}}$.

Similarly, the path-following dynamics $\lambda_{0}$ and the dynamics of the generalized arc-length $\xi_{0}$ for the virtual leader are given as (11) and (13). Without loss of generality, assume the position $p_{0}(t)$ of the virtual leader is on a virtual orbit for all $t \geq 0$, which implies that $\lambda_{0}\left(p_{0}(0)\right)=0, v_{N_{0}}=0, g_{\lambda_{0}} f_{\beta_{N 0}}=0, g_{\xi_{\alpha i}}=0$, and $g_{\xi_{\beta i}} f_{\theta_{i}}=0$. The design for $v_{T_{0}}$ is as follows.

\footnotetext{
*In what follows, to simplify notation, we may use $\theta$ to denote $\theta(t)$.
} 
For $i=1, \cdots, n$, let

$$
\varsigma_{i}=\sum_{j=0}^{n} a_{i j}\left(\xi_{i}-\xi_{j}\right)=a_{i 0}\left(\xi_{i}-\xi_{0}\right)+\sum_{j=1}^{n} a_{i j}\left(\xi_{i}-\xi_{j}\right)
$$

denote the formation errors. The dynamics of $\varsigma_{i}$ are

$$
\dot{\varsigma}_{i}=\sum_{j=0}^{n} a_{i j}\left(\frac{\partial \xi_{i}}{\partial s_{i}} v_{T_{i}}+g_{\xi_{\alpha i}}+g_{\xi_{\beta i}} f_{\theta_{i}} \theta-\frac{\partial \xi_{j}}{\partial s_{j}} v_{T_{j}}-g_{\xi_{\alpha j}}-g_{\xi_{\beta j}} f_{\theta j} \theta\right) .
$$

The equations of the formation-tracking control system consisting of the virtual leader and the followers are given by Eqs. 111, 13, and 15.

\section{B. Controller Design}

We first analyze the simple case of stationary virtual leader and then the case of moving virtual leader. For the virtual leader, the controller $v_{T_{0}}$ is

$$
v_{T_{0}}=0
$$

which yields $\dot{\xi}_{0}=0$. The point on the virtual orbit associated with the motion of the virtual leader is determined therefore by its initial position.

Consider the candidate Lyapunov function

$$
\begin{aligned}
V_{I} & =\sum_{i=1}^{n} \Psi\left(\lambda_{i}\right)+\frac{1}{2} \sum_{i=1}^{n} \varsigma_{i}^{2}+\frac{1}{2 \gamma} \sum_{i=1}^{n}\left(l-\hat{\theta}_{i}\right)^{T}\left(l-\hat{\theta}_{i}\right)+\frac{\rho_{0}}{2 \gamma_{1}} \sum_{i=1}^{n}\left(\rho_{\alpha}-\hat{\rho}_{\alpha_{i}}\right)^{2} \\
& +\frac{\rho_{0}}{2 \gamma_{2}} \sum_{i=1}^{n}\left(\rho_{\beta}-\hat{\rho}_{\beta_{i}}\right)^{2}+\frac{1}{2 \gamma_{3}} \sum_{i=1}^{n}\left(\delta_{*}-\hat{\delta}_{i}\right)^{2}+\frac{1}{2 \gamma_{4}} \sum_{i=1}^{n}\left(\delta_{2 *}-\hat{\delta}_{2 i}\right)^{2},
\end{aligned}
$$

where $l=\left[l_{1}^{T}, \cdots, l_{n}^{T}\right]^{T} \in \mathbb{R}^{n m} . \hat{\rho}_{\alpha_{i}}, \hat{\rho}_{\beta_{i}}, \hat{\delta}_{i}$ and $\hat{\delta}_{2 i}$ are the estimates of $l, \rho_{\alpha}, \rho_{\beta}, \delta_{*}$ and $\delta_{2 *}=\delta_{*}|l|$, respectively. Moreover $\rho_{\alpha}=\rho_{M}^{2} \delta_{*} / \rho_{0}, \rho_{\beta}=\rho_{M}^{2} \delta_{2 *} / \rho_{0}$, where $\rho_{M}$ is the maximum eigenvalue of the matrix $L_{1}^{T} L_{1}$, and $\rho_{0}$ is the minimum eigenvalue of the matrix $\left(L_{1}^{T}+L_{1}\right) / 2$. The constants $\gamma, \gamma_{1}, \gamma_{2}, \gamma_{3}$ and $\gamma_{4}$ are positive adaptation gains.

In the candidate Lyapunov function (17), the first term contributes to achieving the path-following objective, i.e., Eqs. (3) and (4). It vanishes when $\lambda_{i}=0$. The second term contributes to achieving the formation, i.e., the objective expressed by Eq. [12); it vanishes when $\varsigma_{i}=0$. The third term is used to design the parameter update law $\dot{\hat{\theta}}_{i}$ using the congelation of variables method, in which the parameter update law $\dot{\hat{\theta}}_{i}$ is used to estimate an unknown constant vector $l$ instead of $\theta(t)$. The next four terms contribute to achieving the estimation of the eigenvalue-based parameters associated with $L_{1}$ and the estimation of the unknown 
parameters associated with the bound on the time-varying parameters. Differentiating both sides of Eq. 17 along the trajectories of Eqs. 111, 13) and (15) yields

$$
\begin{aligned}
\dot{V}_{I} & =\sum_{i=1}^{n} \nabla \Psi_{i}\left(\left\|\nabla \lambda_{i}\right\| v_{N_{i}}+g_{\lambda_{i}} f_{\beta_{N i}} \theta\right)+\sum_{i=1}^{n} \varsigma_{i} \sum_{j=0}^{n} a_{i j}\left(\frac{\partial \xi_{i}}{\partial s_{i}} v_{T_{i}}+g_{\xi_{\alpha i}}+\left(g_{\xi_{\beta i}} f_{\theta_{i}}-g_{\xi_{\beta j}} f_{\theta_{j}}\right) \theta\right. \\
& \left.-\frac{\partial \xi_{j}}{\partial s_{j}} v_{T_{j}}-g_{\xi_{\alpha j}}\right)-\frac{1}{\gamma} \sum_{i=1}^{n}\left(l-\hat{\theta}_{i}\right)^{T} \dot{\hat{\theta}}_{i}-\frac{\rho_{0}}{\gamma_{1}} \sum_{i=1}^{n}\left(\rho_{\alpha}-\hat{\rho}_{\alpha_{i}}\right) \dot{\hat{\rho}}_{\alpha_{i}}-\frac{\rho_{0}}{\gamma_{2}} \sum_{i=1}^{n}\left(\rho_{\beta}-\hat{\rho}_{\beta_{i}}\right) \dot{\hat{\rho}}_{\beta_{i}} \\
& -\frac{1}{\gamma_{3}} \sum_{i=1}^{n}\left(\delta_{*}-\hat{\delta}_{i}\right) \dot{\hat{\delta}}_{i}-\frac{1}{\gamma_{4}} \sum_{i=1}^{n}\left(\delta_{2 *}-\hat{\delta}_{2 i}\right) \dot{\hat{\delta}}_{2 i} \\
& =\sum_{i=1}^{n} \nabla \Psi_{i}\left(\left\|\nabla \lambda_{i}\right\| v_{N_{i}}+g_{\lambda_{i}} f_{\beta_{N i}}\left(\theta-l+\hat{\theta}_{i}\right)\right) \\
& +\sum_{i=1}^{n} \varsigma_{i}^{n} \sum_{j=0}^{n} a_{i j}\left(\frac{\partial \xi_{i}}{\partial s_{i}} v_{T_{i}}+g_{\xi_{\alpha i}}+\left(g_{\xi_{\beta i}} f_{\theta_{i}}-g_{\xi_{\beta j}} f_{\theta_{j}}\right)\left(\theta-l+\hat{\theta}_{i}\right)-\frac{\partial \xi_{j}}{\partial s_{j}} v_{T_{j}}-g_{\xi_{\alpha j}}\right) \\
& +\sum_{i=1}^{n}\left(l-\hat{\theta}_{i}\right)^{T}\left(-\frac{1}{\gamma} \dot{\hat{\theta}}_{i}+\nabla \Psi_{i} g_{\lambda_{i}} f_{\beta_{N i}}^{T}+\varsigma_{i} \sum_{j=0}^{n} a_{i j}\left(g_{\xi_{\beta i}} f_{\theta_{i}}^{T}-g_{\xi_{\beta j}} f_{\theta_{j}}^{T}\right)\right) \\
& -\frac{\rho_{0}}{\gamma_{1}} \sum_{i=1}^{n}\left(\rho_{\alpha}-\hat{\rho}_{\alpha_{i}}\right) \dot{\hat{\rho}}_{\alpha_{i}}-\frac{\rho_{0}}{\gamma_{2}} \sum_{i=1}^{n}\left(\rho_{\beta}-\hat{\rho}_{\beta_{i}}\right) \dot{\hat{\rho}}_{\beta_{i}}-\frac{1}{\gamma_{3}} \sum_{i=1}^{n}\left(\delta_{*}-\hat{\delta}_{i}\right) \dot{\hat{\delta}}_{i}-\frac{1}{\gamma_{4}} \sum_{i=1}^{n}\left(\delta_{2 *}-\hat{\delta}_{2 i}\right) \dot{\hat{\delta}}_{2 i}
\end{aligned}
$$

consider

$$
\begin{aligned}
v_{N_{i}} & =-\frac{1}{\left\|\nabla \lambda_{i}\right\|}\left(g_{\lambda_{i}} f_{\beta_{N i}} \hat{\theta}_{i}+k_{1} f_{\alpha_{i}}\right), \\
v_{T_{i}} & =\left(\frac{\partial \xi_{i}}{\partial s_{i}}\right)^{-1}\left(-g_{\xi_{\alpha i}}-\sum_{j=0}^{n} a_{i j}\left(g_{\xi_{\beta i}} f_{\theta_{i}}-g_{\xi_{\beta j}} f_{\theta_{j}}\right) \hat{\theta}_{i}-k_{2} \varsigma_{i}\right), \\
\dot{\hat{\theta}}_{i} & =\gamma\left(\nabla \Psi_{i} g_{\lambda_{i}} f_{\beta_{N i}}^{T}+\varsigma_{i} \sum_{j=0}^{n} a_{i j}\left(g_{\xi_{\beta i}} f_{\theta_{i}}^{T}-g_{\xi_{\beta j}} f_{\theta_{j}}^{T}\right)\right)
\end{aligned}
$$

in which the control parameters $k_{1}$ and $k_{2}$ are be selected. Let $\varsigma_{0}=0$ and $\hat{\theta}_{0}=0$, then $v_{T_{i}}$ in Eq. $19 \mathrm{~b}$ is the same as $v_{T_{0}}$ in Eq. 16. Substituting Eq. 19 into Eq. 18 yields

$$
\begin{aligned}
\dot{V}_{I} & =\sum_{i=1}^{n} \nabla \Psi_{i}\left(-k_{1} f_{\alpha_{i}}+g_{\lambda_{i}} f_{\beta_{N i}}(\theta-l)\right)-k_{2} \varsigma^{T} \frac{L_{1}^{T}+L_{1}}{2} \varsigma+\Xi_{1}+\Xi_{2}-\frac{\rho_{0}}{\gamma_{1}} \sum_{i=1}^{n}\left(\rho_{\alpha}-\hat{\rho}_{\alpha_{i}}\right) \dot{\hat{\rho}}_{\alpha_{i}} \\
& -\frac{\rho_{0}}{\gamma_{2}} \sum_{i=1}^{n}\left(\rho_{\beta}-\hat{\rho}_{\beta_{i}}\right) \dot{\hat{\rho}}_{\beta_{i}}-\frac{1}{\gamma_{3}} \sum_{i=1}^{n}\left(\delta_{*}-\hat{\delta}_{i}\right) \dot{\hat{\delta}}_{i}-\frac{1}{\gamma_{4}} \sum_{i=1}^{n}\left(\delta_{2 *}-\hat{\delta}_{2 i}\right) \dot{\hat{\delta}}_{2 i},
\end{aligned}
$$

where $\varsigma=\left[\varsigma_{1}, \cdots, \varsigma_{n}\right]^{T}, \Xi_{1}=\sum_{i=1}^{n} \varsigma_{i} \sum_{j=0}^{n} a_{i j}\left(g_{\xi_{\beta i}} f_{\theta_{i}}-g_{\xi_{\beta j}} f_{\theta_{j}}\right)(\theta-l)$ and $\Xi_{2}=\sum_{i=1}^{n} \varsigma_{i} \sum_{j=0}^{n} a_{i j} \sum_{i=0}^{n} a_{j i}\left(g_{\xi_{\beta j}} f_{\theta_{j}}\right.$ 
$\left.-g_{\xi_{\beta i}} f_{\theta_{i}}\right) \hat{\theta}_{j}$. By definition of $\lambda_{i}$ in [7] and by Assumptions 3 and 4 the inequalities

$$
\begin{aligned}
& \left\|f_{\beta_{N i}}\right\| \leq c_{1},\left\|f_{\beta_{T i}}\right\| \leq c_{1},\left\|\nabla \lambda_{i}\right\| \leq c_{2}, \quad\left|\frac{\partial \xi_{i}}{\partial s_{i}}\right| \leq c_{3}, \quad\left|\frac{\partial s_{i}}{\partial \lambda_{i}}\right| \leq \int_{0}^{2 \pi} \sqrt{\left(\dot{p}_{x_{i}}(\tau)\right)^{2}+\left(\dot{p}_{y_{i}}(\tau)\right)^{2}} d \tau \leq c_{4}, \\
& \left\|\frac{\partial \xi_{i}}{\partial s_{i}} f_{\theta_{i}}\right\| \leq c_{1} c_{3}\left(1+c_{2} c_{4}\right)=c_{5}, \quad\left|g_{\xi_{\beta i}} f_{\theta_{i}}\right| \leq c_{3} c_{5}\left|f_{\alpha_{i}}\right|, \quad|\theta-l| \leq \delta_{*}
\end{aligned}
$$

hold on the set $\Omega_{i}$, for some positive constants $c_{k}, k=1, \cdots, 5$.

Let $\varsigma_{\theta}=\left[\varsigma_{1}(\theta-l), \cdots, \varsigma_{n}(\theta-l)\right]^{T}, g_{\xi_{\beta}}=\left[g_{\xi_{\beta 1}} f_{\theta_{1}}, \cdots, g_{\xi_{\beta n}} f_{\theta_{n}}\right]^{T}$ and $f_{\alpha}=\left[f_{\alpha_{1}}, \cdots, f_{\alpha_{1}}\right]^{T}$ and note that

$$
\Xi_{1}=\Xi_{1}^{T}=\sum_{i=1}^{n} \varsigma_{i}(\theta-l)^{T} \sum_{j=0}^{n} a_{i j}\left(g_{\xi_{\beta i}} f_{\theta_{i}}^{T}-g_{\xi_{\beta j}} f_{\theta_{j}}^{T}\right)
$$

Due to the fact that $g_{\xi_{\beta 0}} f_{\theta_{0}}^{T}=0$ we have

$$
\sum_{j=0}^{n} a_{i j}\left(g_{\xi_{\beta i}} f_{\theta_{i}}^{T}-g_{\xi_{\beta j}} f_{\theta_{j}}^{T}\right)=a_{i 0} g_{\xi_{\beta i}} f_{\theta_{i}}^{T}+\sum_{j=1}^{n} a_{i j}\left(g_{\xi_{\beta i}} f_{\theta_{i}}^{T}-g_{\xi_{\beta j}} f_{\theta_{j}}^{T}\right)=L_{1} \otimes I g_{\xi_{\beta}},
$$

which yields

$$
\Xi_{1}=\varsigma_{\theta}^{T} L_{1} \otimes I g_{\xi_{\beta}}
$$

From Eqs. 210 one has

$$
\varsigma_{\theta}^{T} L_{1} \otimes I g_{\xi_{\beta}} \leq\left\|\varsigma_{\theta}^{T}\right\|\left\|L_{1} \otimes I\right\|\left\|g_{\xi_{\beta}}\right\| \leq \rho_{M}\left\|\varsigma_{\theta}^{T}\right\|\left\|g_{\xi_{\beta}}\right\| \leq c_{3} c_{5} \rho_{M} \delta_{*}\left\|\varsigma^{T}\right\|\left\|f_{\alpha}\right\| .
$$

Finally let $\hat{\theta}_{a_{i}}=a_{j i} \hat{\theta}_{i}^{T}, \hat{\theta}_{a}=\left[\hat{\theta}_{a_{1}}^{T}, \cdots, \hat{\theta}_{a_{n}}^{T}\right]^{T}$ and $\hat{\theta}=\left[\hat{\theta}_{1}^{T}, \cdots, \hat{\theta}_{n}^{T}\right]^{T}$. Then

$$
\Xi_{2}=\sum_{i=0}^{n} a_{j i} \hat{\theta}_{i}^{T} \sum_{j=0}^{n} a_{i j}\left(g_{\xi_{\beta i}} f_{\theta_{i}}^{T}-g_{\xi_{\beta j}} f_{\theta_{j}}^{T}\right)=\sum_{i=1}^{n} a_{j i} \hat{\theta}_{i}^{T} \sum_{j=0}^{n} a_{i j}\left(g_{\xi_{\beta i}} f_{\theta_{i}}^{T}-g_{\xi_{\beta j}} f_{\theta_{j}}^{T}\right)=\hat{\theta}_{a}^{T} L_{1} \otimes I g_{\xi_{\beta}} .
$$

From 21 one has

$$
\hat{\theta}_{a}^{T} L_{1} \otimes I g_{\xi_{\beta}} \leq\left\|\hat{\theta}_{a}^{T}\right\|\left\|L_{1} \otimes I\right\|\left\|g_{\xi_{\beta}}\right\| \leq c_{3} c_{5} \rho_{M}\left\|\hat{\theta}^{T}\right\|\left\|f_{\alpha}\right\|
$$

According to Lemma 2 in 31,

$$
n^{1 / 2}\|\varsigma\|=n^{1 / 2}\left(\sum_{i=1}^{n} \varsigma_{i}^{2}\right)^{1 / 2} \geq \sum_{i=1}^{n}\left|\varsigma_{i}\right|
$$


Exploiting Eqs. 21], 25], 27) and (28) we conclude that

$$
\begin{aligned}
\dot{V}_{I} & \leq-\sum_{i=1}^{n} k_{1} \nabla \Psi_{i} f_{\alpha_{i}}+\sum_{i=1}^{n} c_{1} c_{2} \delta_{*}\left|\nabla \Psi_{i} f_{\alpha_{i}}\right|-k_{2} \varsigma^{T} \frac{L_{1}^{T}+L_{1}}{2} \varsigma+c_{3} c_{5} \rho_{M} \delta_{*}\left\|\varsigma^{T}\right\|\left\|f_{\alpha}\right\| \\
& +n^{1 / 2} c_{3} c_{5} \rho_{M} \delta_{*}\left\|\hat{\theta}^{T}\right\|\left\|f_{\alpha}\right\|\|\varsigma\|-\frac{\rho_{0}}{\gamma_{1}} \sum_{i=1}^{n}\left(\rho_{\alpha}-\hat{\rho}_{\alpha_{i}}\right) \dot{\hat{\rho}}_{\alpha_{i}}-\frac{\rho_{0}}{\gamma_{2}} \sum_{i=1}^{n}\left(\rho_{\beta}-\hat{\rho}_{\beta_{i}}\right) \dot{\hat{\rho}}_{\beta_{i}}-\frac{1}{\gamma_{3}} \sum_{i=1}^{n}\left(\delta_{*}-\hat{\delta}_{i}\right) \dot{\hat{\delta}}_{i} \\
& -\frac{1}{\gamma_{4}} \sum_{i=1}^{n}\left(\delta_{2 *}-\hat{\delta}_{2 i}\right) \dot{\hat{\delta}}_{2 i} .
\end{aligned}
$$

On the set $\Phi_{I}=\left\{\left(\lambda_{i}, \varsigma_{i}, l-\hat{\theta}_{i}, \rho_{\alpha}-\hat{\rho}_{\alpha_{i}}, \rho_{\beta}-\hat{\rho}_{\beta_{i}}, \delta_{*}-\hat{\delta}_{i}, \delta_{2 *}-\hat{\delta}_{2 i}\right) \mid V_{I} \leq c_{6}\right\}$, for some $c_{6}>0$, one has

$$
\left|\hat{\theta}_{i}\right| \leq\left|l-\hat{\theta}_{i}\right|+|l| \leq \sqrt{2 \gamma c_{6}}+|l|=c_{7}+|l|
$$

By applying Young's inequality

$$
\|\varsigma\|\left\|f_{\alpha}\right\|=\left(\sum_{i=1}^{n} \varsigma_{i}^{2}\right)^{1 / 2}\left(\sum_{i=1}^{n} f_{\alpha_{i}}^{2}\right)^{1 / 2} \leq \frac{\rho_{M}}{2} \sum_{i=1}^{n} \varsigma_{i}^{2}+\frac{1}{2 \rho_{M}} \sum_{i=1}^{n} f_{\alpha_{i}}^{2}
$$

and Eq. (30) to Eq. 290 yields

$$
\begin{aligned}
\dot{V}_{I} & \leq-\left(k_{1}-\left(c_{1} c_{2}-\frac{1}{2} c_{3} c_{5}\left(1+n^{1 / 2} c_{7}\right)\right) \hat{\delta}_{*}-\frac{1}{2} c_{3} c_{5} n^{1 / 2} \hat{\delta}_{2 *}\right) \sum_{i=1}^{n} \nabla \Psi_{i} f_{\alpha_{i}}-\frac{1}{2} c_{3} c_{5} \delta_{*}\left(1+n^{1 / 2}\left(c_{7}+|l|\right)\right) \\
& \times \sum_{i=1}^{n}\left(\nabla \Psi_{i} f_{\alpha_{i}}-f_{\alpha_{i}}^{2}\right)-\rho_{0}\left(k_{2}-\frac{1}{2} c_{3} c_{5}\left(1+n^{1 / 2} c_{7}\right) \hat{\rho}_{\alpha_{i}}-\frac{1}{2} c_{3} c_{5} n^{1 / 2} \hat{\rho}_{\beta_{i}}\right) \sum_{i=1}^{n} \varsigma_{i}^{2} \\
& +\rho_{0} \sum_{i=1}^{n}\left(\rho_{\alpha}-\hat{\rho}_{\alpha_{i}}\right)\left(-\frac{1}{\gamma_{1}} \dot{\hat{\rho}}_{\alpha_{i}}+\frac{1}{2} c_{3} c_{5}\left(1+n^{1 / 2} c_{7}\right) \varsigma_{i}^{2}\right)+\rho_{0} \sum_{i=1}^{n}\left(\rho_{\beta}-\hat{\rho}_{\beta_{i}}\right)\left(-\frac{1}{\gamma_{2}} \dot{\hat{\rho}}_{\beta_{i}}+\frac{1}{2} c_{3} c_{5} n^{1 / 2} \varsigma_{i}^{2}\right) \\
& +\sum_{i=1}^{n}\left(\delta_{*}-\hat{\delta}_{i}\right)\left(-\frac{1}{\gamma_{3}} \dot{\hat{\delta}}_{i}+\left(c_{1} c_{2}-\frac{1}{2} c_{3} c_{5}\left(1+n^{1 / 2} c_{7}\right)\right) \nabla \Psi_{i} f_{\alpha_{i}}\right) \\
& +\sum_{i=1}^{n}\left(\delta_{2 *}-\hat{\delta}_{2 i}\right)\left(-\frac{1}{\gamma_{4}} \dot{\hat{\delta}}_{2 i}+\frac{1}{2} c_{3} c_{5} n^{1 / 2} \nabla \Psi_{i} f_{\alpha_{i}}\right) .
\end{aligned}
$$

Let

$$
\begin{aligned}
k_{1} & =\left(c_{1} c_{2}-\frac{1}{2} c_{3} c_{5}\left(1+n^{1 / 2} c_{7}\right)\right) \hat{\delta}_{*}+\frac{1}{2} c_{3} c_{5} n^{1 / 2} \hat{\delta}_{2 *}+\epsilon_{1} \\
k_{2} & =\frac{1}{2} c_{3} c_{5}\left(1+n^{1 / 2} c_{7}\right) \hat{\rho}_{\alpha_{i}}+\frac{1}{2} c_{3} c_{5} n^{1 / 2} \hat{\rho}_{\beta_{i}}+\epsilon_{2}, \\
\dot{\hat{\rho}}_{\alpha_{i}} & =\frac{1}{2} \gamma_{1} c_{3} c_{5}\left(1+n^{1 / 2} c_{7}\right) \varsigma_{i}^{2}, \\
\dot{\hat{\rho}}_{\beta_{i}} & =\frac{1}{2} \gamma_{2} c_{3} c_{5} n^{1 / 2} \varsigma_{i}^{2}
\end{aligned}
$$




$$
\begin{aligned}
\dot{\hat{\delta}}_{i} & =\gamma_{3}\left(c_{1} c_{2}-\frac{1}{2} c_{3} c_{5}\left(1+n^{1 / 2} c_{7}\right)\right) \nabla \Psi_{i} f_{\alpha_{i}}, \\
\dot{\hat{\delta}}_{2 i} & =\frac{1}{2} \gamma_{4} c_{3} c_{5} n^{1 / 2} \nabla \psi_{i} f_{\alpha_{i}},
\end{aligned}
$$

with $\epsilon_{1}$ and $\epsilon_{2}$ positive constants. Substituting Eqs. (33) into Eq. 32 yields

$$
\dot{V}_{I} \leq W_{I}
$$

where

$$
W_{I}=-\epsilon_{1} \sum_{i=1}^{n} \nabla \Psi_{i} f_{\alpha_{i}}-\rho_{0} \epsilon_{2} \sum_{i=1}^{n} \varsigma_{i}^{2}
$$

Recalling Assumptions 1 and 2, Lemma 1 and Eq. (33), we conclude that $W_{I} \leq 0$ hence $\dot{V}_{I} \leq 0$. Note now that, by Eq. (19), the closed-loop system associated to the formation tracking control system for the $i$ th follower is

$$
\begin{aligned}
& \dot{\lambda}_{i}=-k_{1} f_{\alpha_{i}}+g_{\lambda_{i}} f_{\beta_{N i}}\left(\theta-\hat{\theta}_{i}\right), \\
& \dot{\zeta}_{i}=\sum_{j=0}^{n} a_{i j}\left(-k_{2}\left(\varsigma_{i}-\varsigma_{j}\right)+\left(g_{\xi_{\beta i}} f_{\theta_{i}}-g_{\xi_{\beta j}} f_{\theta_{j}}\right)\left(\theta-\hat{\theta}_{i}\right)-\sum_{i=0}^{n} a_{j i}\left(g_{\xi_{\beta j}} f_{\theta_{j}}-g_{\xi_{\beta i}} f_{\theta_{i}}\right) \hat{\theta}_{j}\right), \\
& \dot{\hat{\theta}}_{i}=\gamma\left(\nabla \Psi_{i} g_{\lambda_{i}} f_{\beta_{N i}}^{T}+\varsigma_{i} \sum_{j=0}^{n} a_{i j}\left(g_{\xi_{\beta i}} f_{\theta_{i}}^{T}-g_{\xi_{\beta j}} f_{\theta_{j}}^{T}\right)\right),
\end{aligned}
$$

with the control gains $k_{1}$ and $k_{2}$ satisfying conditions (33).

In the following we will consider the case of the moving virtual leader. The control $v_{T_{0}}$ for the moving virtual leader is set as

$$
\begin{aligned}
v_{T_{0}} & =-\left(\frac{\partial \xi_{0}}{\partial s_{0}}\right)^{-1} k_{2} \varsigma_{0} \\
\dot{\varsigma}_{0} & =-k_{2} \sum_{i=1}^{n} a_{i 0} \varsigma_{i}+\eta_{*}(t),
\end{aligned}
$$

where $\varsigma_{0}$ is used to adjust the speed of the virtual leader to maintain the formation performance. $\eta_{*}(t) \in \mathbb{R}$ is a bounded signal, which forces the virtual leader to move along its virtual orbit at a specific orbital speed. The control term $k_{2} \varsigma_{0}$ in the first of equations of 36 is a formation adjustment unit to keep the formation accuracy of the whole formation. If we let $\eta_{*}(t)=0, \varsigma_{0}(0)=0$ and $k_{2}=0$, the control law (36) for the moving virtual leader is the same as the one in Eq. 16) for the stationary virtual leader.

Remark 6. A similar idea has been proposed in [32, in which a function of the error between the state 
of the $i$ th reference node and the state of the $i$ th agent is added into the instantiation dynamics of the $i$ th reference node in the virtual structure. It is indicated therein that such additional function can improve the consensus accuracy but a specific form was not given.

Remark \%. When the formation objective is achieved, one finds that $\varsigma_{i}=0$ and then $\dot{\varsigma}_{0}=\eta_{*}$ as $t \rightarrow \infty$. Note that $\dot{\xi}_{0}=-k_{2} \varsigma_{0}$. As a result, the virtual leader and all the followers move along their desired orbits with the given speed.

Consider now the candidate Lyapunov function

$$
V_{I I}=V_{I}+\frac{1}{2}\left(\varsigma_{0}-\int_{0}^{t} \eta_{*}(\tau) d \tau\right)^{2}
$$

Differentiating both sides of Eq. 37 along $v_{N_{i}}, v_{T_{i}}$ and $\dot{\hat{\theta}}_{i}$ as in Eqs. 19 yields

$$
\begin{aligned}
\dot{V}_{I I} & =\sum_{i=1}^{n} \nabla \Psi_{i}\left(-k_{1} f_{\alpha_{i}}+g_{\lambda_{i}} f_{\beta_{N i}}(\theta-l)\right)+\left(\varsigma_{0}-\int_{0}^{t} \eta_{*}(\tau) d \tau\right)\left(\dot{\varsigma}_{0}-\eta_{*}\right) \\
& +\sum_{i=1}^{n} \varsigma_{i} \sum_{j=0}^{n} a_{i j}\left(-k_{2}\left(\varsigma_{i}-\varsigma_{j}\right)+\left(g_{\xi_{\beta i}} f_{\theta_{i}}-g_{\xi_{\beta j}} f_{\theta_{j}}\right)(\theta-l)-\sum_{i=0}^{n} a_{j i}\left(g_{\xi_{\beta j}} f_{\theta_{j}}-g_{\xi_{\beta i}} f_{\theta_{i}}\right) \hat{\theta}_{j}\right) \\
& -\frac{\rho_{0}}{\gamma_{1}} \sum_{i=1}^{n}\left(\rho_{\alpha}-\hat{\rho}_{\alpha_{i}}\right) \dot{\hat{\rho}}_{\alpha_{i}}-\frac{\rho_{0}}{\gamma_{2}} \sum_{i=1}^{n}\left(\rho_{\beta}-\hat{\rho}_{\beta_{i}}\right) \dot{\hat{\rho}}_{\beta_{i}}-\frac{1}{\gamma_{3}} \sum_{i=1}^{n}\left(\delta_{*}-\hat{\delta}_{i}\right) \dot{\hat{\delta}}_{i}-\frac{1}{\gamma_{4}} \sum_{i=1}^{n}\left(\delta_{2 *}-\hat{\delta}_{2 i}\right) \dot{\hat{\delta}}_{2 i} \\
& =\sum_{i=1}^{n} \nabla \Psi_{i}\left(-k_{1} f_{\alpha_{i}}+g_{\lambda_{i}} f_{\beta_{N i}}(\theta-l)\right)+\left(\varsigma_{0}-\int_{0}^{t} \eta_{*}(\tau) d \tau\right)\left(\dot{\varsigma}_{0}-\eta_{*}+k_{2} \sum_{i=1}^{n} a_{i 0} \varsigma_{i}\right) \\
& -k_{2} \varsigma^{T} \frac{L_{1}^{T}+L_{1}}{2} \varsigma+\sum_{i=1}^{n} \varsigma_{i} \sum_{j=0}^{n} a_{i j}\left(\left(g_{\xi_{\beta i}} f_{\theta_{i}}-g_{\xi_{\beta j}} f_{\theta_{j}}\right)(\theta-l)-\sum_{i=0}^{n} a_{j i}\left(g_{\xi_{\beta j}} f_{\theta_{j}}-g_{\xi_{\beta i}} f_{\theta_{i}}\right) \hat{\theta}_{j}\right) \\
& -\frac{\rho_{0}}{\gamma_{1}} \sum_{i=1}^{n}\left(\rho_{\alpha}-\hat{\rho}_{\alpha_{i}}\right) \dot{\hat{\rho}}_{\alpha_{i}}-\frac{\rho_{0}}{\gamma_{2}} \sum_{i=1}^{n}\left(\rho_{\beta}-\hat{\rho}_{\beta_{i}}\right) \dot{\hat{\rho}}_{\beta_{i}}-\frac{1}{\gamma_{3}} \sum_{i=1}^{n}\left(\delta_{*}-\hat{\delta}_{i}\right) \dot{\hat{\delta}}_{i}-\frac{1}{\gamma_{4}} \sum_{i=1}^{n}\left(\delta_{2 *}-\hat{\delta}_{2 i}\right) \dot{\hat{\delta}}_{2 i} .
\end{aligned}
$$

Substituting Eqs. (36) into Eq. (38) and applying a similar analysis to that of the case of the stationary virtual leader yield $\dot{V}_{I I} \leq W_{I} \leq 0$, from which we obtain the following result.

Theorem 1. Suppose that the initial position of each vehicle is such that $p_{i}(0) \in \Omega_{i}$. Assume moreover that Assumptions 1 to 4 hold. Then, for $l=0, \cdots, n$, the formation-tracking problem with a virtual leader is solved by the formation tracking control

$$
u_{l}=\left[\begin{array}{c}
N_{l}^{T} \\
T_{l}^{T}
\end{array}\right]^{-1}\left[\begin{array}{l}
v_{N_{l}} \\
v_{T_{l}}
\end{array}\right],
$$

where $v_{N_{0}}=0, v_{T_{0}}$ is given in 36 and $v_{N_{i}}, v_{T_{i}}$ and $\dot{\hat{\theta}}_{i}$ are as given in Eqs. 19 .

Proof. Let $p_{i}(0) \in \Omega_{i}$ and note that the set $\Phi_{I I}=\left\{\left(\lambda_{i}, \varsigma_{i}, l-\hat{\theta}_{i}, \rho_{\alpha}-\hat{\rho}_{\alpha_{i}}, \rho_{\beta}-\hat{\rho}_{\beta_{i}}, \delta_{*}-\hat{\delta}_{i}, \delta_{2 *}-\hat{\delta}_{2 i}, \varsigma_{0}-\right.\right.$ 
$\left.\left.\int_{0}^{t} \eta_{*}(\tau) d \tau\right) \mid V_{I I} \leq c_{6}\right\}$ is closed by continuity. Note now that $\left|\lambda_{i}\right|<\varepsilon_{i},\left|\varsigma_{i}\right| \leqslant \sqrt{2 c_{6}},\left|l-\hat{\theta}_{i}\right| \leqslant \sqrt{2 c_{6} \gamma}$, $\left|\rho_{\alpha}-\hat{\rho}_{\alpha_{i}}\right| \leqslant \sqrt{2 c_{6} \gamma_{1}},\left|\rho_{\beta}-\hat{\rho}_{\beta_{i}}\right| \leqslant \sqrt{2 c_{6} \gamma_{2}},\left|\delta_{*}-\hat{\delta}_{i}\right| \leqslant \sqrt{2 c_{6} \gamma_{3}},\left|\delta_{2 *}-\hat{\delta}_{2 i}\right| \leqslant \sqrt{2 c_{6} \gamma_{4}}$ and $\left|\varsigma_{0}-\int_{0}^{t} \eta_{*}(\tau) d \tau\right| \leqslant$ $\sqrt{2 c_{6}}$. Thus the set $\Phi_{I I}$ is compact. As a result the closed-loop system 35 is Lipschitz continuous on the set $\Phi_{I I}$ and also piecewise continuous in $t$, which implies that solutions exist and are unique.

Note now that the value of $V_{I I}$ is non-increasing along the trajectories of the system. When the initial value of $V_{I I}$ is finite, the solution stays in $\Phi_{I I}$, which implies that $\left|\lambda_{i}\left(p_{i}(t)\right)\right|<\varepsilon_{i}$ by the definition of the barrier function $\Psi_{i}$ in [29]. By the LaSalle-Yoshizawa Theorem cite, we have that

$$
\lim _{t \rightarrow+\infty} W_{I}(t)=0
$$

that is

$$
\lim _{t \rightarrow+\infty} \nabla \Psi_{i}(t) f_{\alpha_{i}}\left(\lambda_{i}(t)\right)=0
$$

and

$$
\lim _{t \rightarrow+\infty} \varsigma_{i}(t)=\lim _{t \rightarrow \infty} \sum_{j=0}^{n} a_{i j}\left(\xi_{i}-\xi_{j}\right)=0 .
$$

From Eq. 40, and Assumption 2 we therefore conclude that $\lim _{t \rightarrow+\infty} \lambda_{i}(t)=0$. Moreover condition (41) and Assumption 1 imply that condition (6) is satisfied.

Remark 8. The proof of Lemma 1 in [7] yields $\left\|\nabla \lambda_{i}\right\|=\left\|\frac{\partial \lambda_{i}}{\partial p_{i}}\right\| \neq 0$, for all $p_{i} \in \Omega_{i}$. Reviewing the proof of Theorem 1 we conclude that $\left|\lambda_{i}\left(p_{i}(t)\right)\right|<\varepsilon_{i}$, which implies $p_{i}(t) \in \Omega_{i}$, for all $t \geq 0$. On the set $\Phi_{I I}$ the

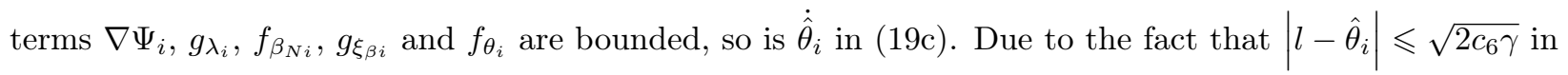
the set $\Phi_{I I}$, and $l$ is a constant vector, we conclude that $\hat{\theta}_{i}$ is also bounded. Note that the definition of the generalized arc-lengths yields $\left|\partial \xi_{i} / \partial s_{i}\right| \geq \varepsilon>0$. Therefore, the control law $(19)$ is well defined.

Remark 9. Reviewing the proof of Theorem 1 we observe that the closed-loop solutions stay in $\Phi_{I I}$. Due to the fact that $\left|\rho_{\alpha}-\hat{\rho}_{\alpha_{i}}\right| \leqslant \sqrt{2 c_{6} \gamma}$ in the set $\Phi_{I I}$ and $\rho_{\alpha}=\rho_{M}^{2} \delta_{*} / \rho_{0}$ is a constant we can conclude that $\hat{\rho}_{\alpha_{i}}$ is bounded. The same applies to $\hat{\rho}_{\beta_{i}}, \hat{\delta}_{i}$ and $\hat{\delta}_{2 i}$. As a result, the control gains $k_{1}$ and $k_{2}$ are bounded.

Remark 10. From Eqs. (35) we conclude that the convergence rates of the states of the resulting path following subsystem and of the resulting formation subsystem depends on the control gains $k_{1}$ and $k_{2}$. The convergence rates of the systems with states $\hat{\theta}_{i}, \hat{\rho}_{\alpha_{i}}, \hat{\rho}_{\beta_{i}}, \hat{\delta}_{i}$ and $\hat{\delta}_{2 i}$ depend on the adaptation gains $\gamma, \gamma_{1}, \gamma_{2}$, $\gamma_{3}$ and $\gamma_{4}$, respectively. To speed up the convergence rates one can increase the gains. However, the increase in gains results in increased control energy and potential lack of noise attenuation.

Remark 11. From Eqs. 19 we conclude that the control law contains only $\varsigma_{i}$, but not $\dot{\varsigma}_{i}$. $\dot{\varsigma}_{i}$ is used during the analysis. Note that $\varsigma_{i}$ in Eq. (14) only depends on the $\xi_{i}$, the state of the agent itself, and on $\xi_{j}$, the neighboring states.

Remark 12. The states and the estimate of the $i$ th vehicle and its neighboring states $\xi_{j}$ and $g_{\xi_{\beta j}} f_{\theta_{j}}$ are 


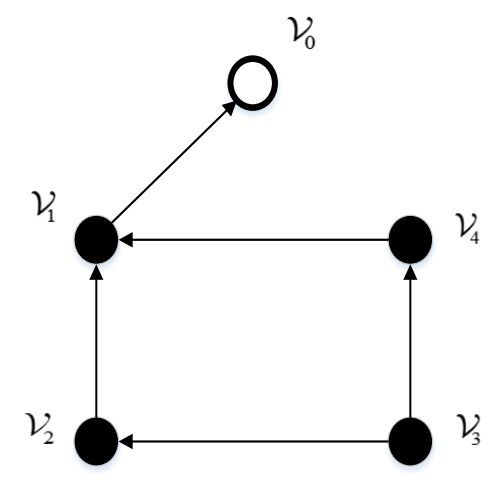

Fig. 2 Digraph illustrating the connections between the vehicles.

used to design the control law 19 . Hence our proposed control law is distributed.

\section{Simulation Results}

The directed network among vehicles is shown in Figure 1. The selected trajectories for the vehicles are concentric ellipses with different semi-major axis and semi-minor axis, that is,

$$
C_{l 0}:\left(\frac{p_{x_{l}}}{e_{l} a}\right)^{2}+\left(\frac{p_{y_{l}}}{e_{l} b}\right)^{2}=1
$$

where $e_{l}=1+0.5(l-1), a=3, b=2, l=0,1,2,3,4$. For $i=1, \cdots, 4$ the effect of the time-varying flowfield is described by

$$
\begin{aligned}
& f_{i}\left(p_{i}, t,\right)=f_{\alpha_{i}}\left(\lambda_{i}\right) f_{\beta_{i}}\left(p_{i}, t\right) \theta_{i}(t) \\
& =\lambda_{i}\left[\begin{array}{cc}
(\cos (0.1+0.01(i-1)) t) & 0 \\
0 & \sin ((0.1+0.01(i-1)) t)
\end{array}\right]\left[\begin{array}{c}
\sin ((0.1+0.01(i-1)) t) \\
\cos ((0.2+0.01(i-1)) t)
\end{array}\right] .
\end{aligned}
$$

The parameters in this case have been selected as $k_{1}=50+10 \hat{\delta}_{*}+10 \hat{\delta}_{2 *}, k_{2}=50+10 \hat{\rho}_{\alpha_{i}}+10 \hat{\rho}_{\beta_{i}}$ and $\gamma=\gamma_{1}=\gamma_{2}=\gamma_{3}=\gamma_{4}=1$. In these simulations the desired formation is given by an in-line pattern and thus the generalized arc-lengths $\xi_{l}$ are selected as $\xi_{l}=s_{l} / e_{l}$.

Case 1: The bounded signal for the virtual leader is $\eta_{*}=0.1$. The motion of the vehicles is illustrated in Figure 3a, where $o, \square,+$ and $\star$ denote the vehicles' positions at $t=0, t=5, t=15$ and $t=20$, respectively. From this figure one can see that the four vehicles converge to the given orbits, form the desired formation and moves along the given orbits. The time histories of the path following errors $\lambda_{i}$ and of the formation errors $\xi_{i}-\xi_{0}$ are plotted in Figures 3b and 3c, respectively. The time histories of the control inputs $v_{N_{i}}$ and $v_{T_{i}}$ are plotted in Figures 3d. Consistently with Theorem 1, path following and formation tracking are achieved. 


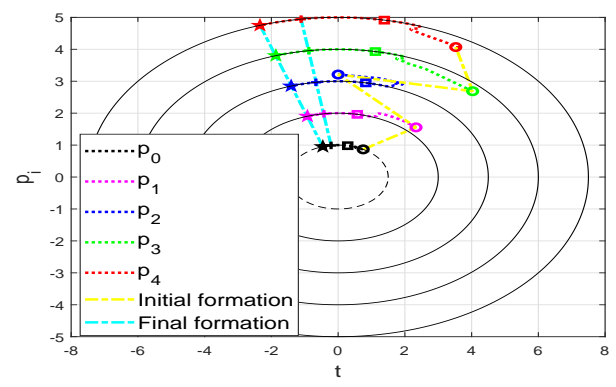

(a)



(c)

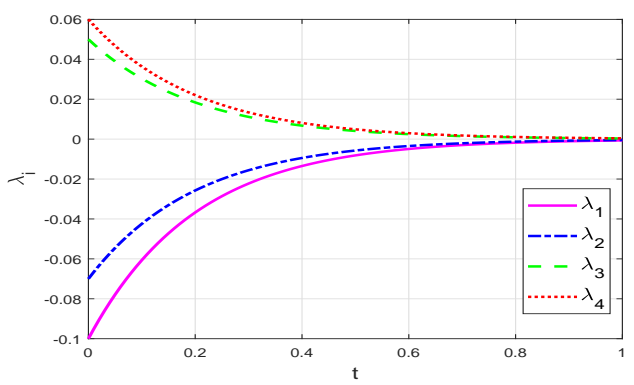

(b)


(d)

Fig. 3 Formation tracking motion with a moving virtual leader.

Case 2: The performance in the case of input saturation $\operatorname{sat}\left(v_{i}(t)\right)=\left[\operatorname{sat}\left(v_{N_{i}}(t)\right), \operatorname{sat}\left(v_{T_{i}}(t)\right)\right]^{T}$, where $\operatorname{sat}\left(v_{N_{i}}(t)\right)=\operatorname{sign}\left(v_{N_{i}}(t)\right) \min \left\{\left|v_{N_{i}}\right|, 100\right\}$ and $\operatorname{sat}\left(v_{T_{i}}(t)\right)=\operatorname{sign}\left(v_{T_{i}}(t)\right) \min \left\{\left|v_{N_{i}}\right|, 100\right\}$, is now discussed. The motion of the vehicles, the path following errors $\lambda_{i}$, the formation errors $\xi_{i}-\xi_{0}$ and the control inputs $v_{N_{i}}$ and $v_{T_{i}}$ are displayed in Figures $4 \mathrm{a}-4 \mathrm{~d}$, where $o, \square,+$ and $\star$ denote the vehicles' positions at $t=0$, $t=5, t=15$ and $t=20$, respectively. Compared to Case 1 the saturated inputs continue to achieve the control task and its performance remains of a good level.

\section{Conclusion}

An adaptive method to solve the formation tracking control problem in the presence of a flowfield with time-varying flow parameters has been presented. First, the case of a stationary virtual leader with unidirectional connected followers is considered and the formation tracking control law is designed by using the congelation of variables method. In order to avoid the use of the eigenvalues of the Laplacian matrix and a bound on the time-varying flow parameters in the control law design, adaptive estimations are designed. By designing the formation adjustment unit for the virtual leader the proposed control can be extended to the case of moving virtual leader. In future work the leaderless formation tracking problem for directed networked vehicles will be considered. 


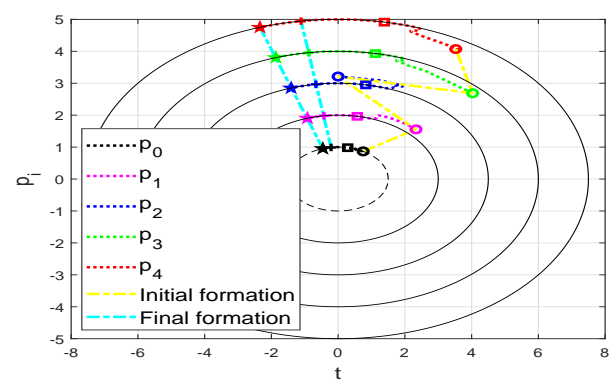

(a)



(c)



(b)
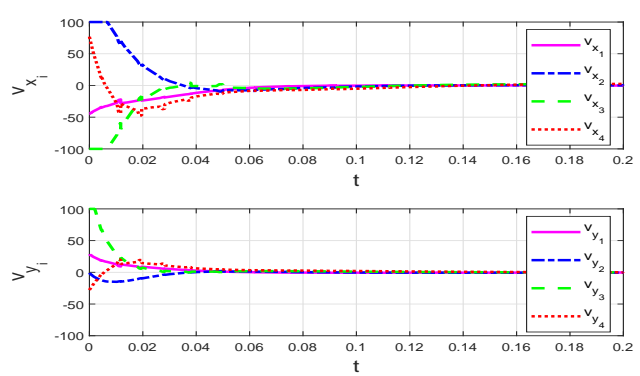

(d)

Fig. 4 Formation tracking motion with saturation inputs.

\section{Acknowledgments}

This work has been partially supported by by the National Natural Science Foundation of China under Grant 61673106, the European Union's Horizon 2020 Research and Innovation Programme under grant agreement No 739551 (KIOS CoE), and by Italian Ministry for Research in the framework of the 2017 Program for Research Projects of National Interest (PRIN), Grant no. 2017YKXYXJ.

\section{References}

[1] Gurfil, P., Idan, M. and Kasdin, N.J., "Adaptive Neural Control of Deep-Space Formation Flying," Journal of Guidance, Control, and Dynamics Vol. 26, No. 3, 2003, pp. 491-501.

doi: $10.2514 / 2.5072$

[2] Bertozzi, A.L., Kemp, M., Marthaler, D., "Determing Enviromental Boundaries: Asynchronous Communication and Physical Scales," Cooperative Control. Lecture Notes in Control and Information Sciences, $1^{\text {st }}$ ed., vol. 309 , V. Kumar, N. Leonard, A.S. Morse, Ed. Germany: Springer, 2005, pp. 25-42.

doi:10.1007/978-3-540-31595-7_2

[3] Fiorelli, E., Leonard, N.E., Vhatta, P., Paley, D.A., Bachmayer, R., Fratantoni, D.M., "Multi-AUV Control and Adaptive Sampling in Monterey Bay," IEEE Journal of Oceanic Engineering, Vol. 31, No. 4, 2006, pp. 935-948. doi:10.1109/JOE.2006.880429 
[4] Ren, W. and Beard, R.W., "Decentralized Scheme for Spacecraft Formation Flying Via the Virtual Structure Approach," Journal of Guidance, Control, and Dynamics, Vol. 27, No. 1, 2004, pp. 73-82. doi: $10.2514 / 1.9287$

[5] Ghabcheloo, R., "Coordinated Path Following of Multiple Autonomous Vehicles," Ph.D. Dissertation, Instituto Superior Tećnico, Lisbon University of Lisbon, Portugal, 2007.

[6] Zhang F. and Leonard, N.E., "Coordinated Patterns of Unit Speed Particles on a Closed Curve," Systems \& Control Letters, Vol. 56, No. 6, 2007, pp. 397-407.

doi:10.1016/j.sysconle.2006.10.027

[7] Chen, Y.-Y. and Tian, Y.-P., "Formation Tracking and Attitude Synchronization Control of Underactuated Ships Along Closed Orbits," International Journal of Robust and Nonlinear Control, Vol. 25, No. 16, 2015, pp. 3023-3044. doi:10.1002/rnc.3246

[8] Leonard, N.E., Paley, D.A., Lekien, F., Sepulchre, R., Fratantoni, D.M., Davis, R.E., "Collective Motion, Sensor Networks, and Ocean Sampling," Proceedings of the IEEE, Vol. 95, No. 1, 2007, pp. 48-74. doi:10.1109/JPROC.2006.887295

[9] Paley, D.A. and Peterson, C., "Stabilization of Collective Motion in a Time-Invariant Flowfield," Journal of Guidance, Control, and Dynamics, Vol. 32, No. 3, 2009, pp. 771-779.

doi:10.2514/1.40636

[10] Peterson, C. and Paley, D.A., "Multivehicle Coordination in an Estimated Time-Varying Flowfield," Journal of Guidance, Control, and Dynamics, Vol. 34, No. 1, 2011, pp. 177-191.

doi: $10.2514 / 1.50036$

[11] C. K. Peterson and D. A. Paley, "Distributed Estimation for Motion Coordination in an Unknown Spatially Varying Flowfield," Journal of Guidance, Control, and Dynamics, Vol. 36, No. 3, 2013, pp. 894-898. doi:10.2514/1.59453

[12] Peng, Z., Wang, D., Wang, H., Wang, W., "Coordinated Formation Pattern Control of Multiple Marine Surface Vehicles with Model Uncertain and Time-Varying Ocean Currents," Neural Computing and Applications, Vol. 25, No. 7-6, 2014, pp. 1771-1783.

doi:10.1007/s00521-014-1668-z

[13] Chen, Y.-Y., Zhang, Y., Wang, Z.-Z. "An Adaptive Backstepping Design for Formation Tracking Motion in an Unknown Eulerian Specification Flowfield," Journal of the Franklin Institute, Vol. 354, No. 14, 2017, pp. 6217-6233. doi:10.1016/j.jfranklin.2017.07.020

[14] Yu, H. and Xia, X., "Adaptive Consensus of Multi-Agents in Networks with Jointly Connected Topologies," Automatica, Vol. 48, No. 8, 2015, pp. 1783-1790.

doi:10.1016/j.automatica.2012.05.068 
[15] Wang, X., Li, S., Lam, J., "Distributed Active Anti-Disturbance Output Consensus Algorithms for Higher-Order Multi-Agent Systems with Mismatched Disturbances," Automatica, Vol. 71, 2016, pp. 30-37. doi:10.1016/j.automatica.2016.07.010

[16] Ghapani, S., Rahili, S., Ren, W., "Distributed Average Tracking of Physical Second-Order Agents With Heterogeneous Unknown Nonlinear Dynamics with Contraint on Input Signals," IEEE Transactions on Automatic Control, Vol. 64, No. 3, 2019, pp. 1178-1184.

doi:10.1109/TAC.2018.2840452

[17] Ren, W. and Atkins, E, "Distributed Multi-Vehicle Coordinated Control via Local Information Exchange," International Journal of Robust and Nonlinear Control, Vol. 17, No. 10-11, 2007, pp. 1002-1033. doi:10.1002/rnc.1147

[18] Leonard, J.J. and Durrant-Whyte, H.F., "Mobile Robot Localization by Tracking Geometric Beacons," IEEE Transactions on Robotics and Automation Vol. 7, No. 3, 1991, pp. 376-382.

doi:10.1109/70.88147

[19] Guo, J., Yan, G., Lin, Z., "Local Control Strategy for Moving-Target-Enclosing Under Dynamically Changing Network Topology," Systems $\&$ Control Letters, Vol. 59, No. 10, 2010, pp. 654-661. doi:10.1016/j.sysconle.2010.07.010

[20] Chen, Y.-Y., Ai, X., Zhang, Y., "Spherical Formation Tracking Control for Second-Order Agents with Unknown General Flowfields and Strongly Connected Topologies," International Journal of Robust and Nonlinear Control, Vol. 29, No. 11, 2019, pp. 3715-3735.

doi:10.1002/rnc.4576

[21] Liu, L., Wang, D., Peng, Z., Chen, C.L.P., Li, T., Lan, W., "Bounded Neural Network Control for Target Tracking of Underactuated Autonomous Surface Vehicles in the Presence of Uncertain Target Dynamics," IEEE Transactions on Neural Networks and Learning Systems, Vol. 30, No. 4, 2019, pp. 1241-1249.

doi:10.1109/TNNLS.2018.2868978

[22] Su, Y. and Huang. J., "Cooperative Output Regulation with Application to Multi-Agent Consensus under Switching Network," IEEE Transactions on Systems, Man, and Cybernetics, Part B (Cybernetics), Vol. 42, No. 3, 2012, pp. 864-875.

doi:10.1109/TSMCB.2011.2179981

[23] Liu, W. and Huang, J., "Adaptive Leader-Following Consensus for a Class of Higher-Order Nonlinear Multi-Agent System with Directed Switching Networks," Automatica, Vol. 79, 2017, pp. 84-92.

doi:10.1016/j.automatica.2017.02.010

[24] Lynch, K.M., Schwartz, I.B., Yang, P., Freeman, R.A., 'Decentralized Environmental Modeling by Mobile Sensor Networks," IEEE Transaction on Robotics, Vol. 24, No. 3, 2008, pp. 710-724.

doi:10.1109/TRO.2008.921567 
[25] Chen, K. and Astolfi, A., "Adaptive Control of Linear Systems with Time-Varying Parameters," American Control Conference, IEEE Publications, Milwaukee, WI, USA, 2018, pp. 80-85.

doi:10.23919/ACC.2018.8431444

[26] Chen, K. and Astolfi, A., "I\&I Adaptive Control for Systems with Varying Parameters," IEEE Conference on Decision and Control, IEEE Publications, Fl, USA, 2018, pp. 2205-2210.

doi:10.1109/CDC.2018.8619564

[27] Chen, K. and Astolfi, A., "Output-Feedback Adaptive Control for Systems With Time-Varying Parameters," IFAC-PapersOnLine, Vol. 52, No. 16, , 2019, pp. 586-591.

doi:10.1016/j.ifacol.2019.12.025

[28] Chen, K. and Astolfi, A., "Adaptive Control for Systems with Time-Varying Parameters," IEEE Transactions on Automatic Control, available online, 2021.

doi:10.1109/TAC.2020.3046141

[29] Chen, Y.-Y., Chen, K., Astolfi, A., "Adaptive Formation Tracking Control for First-Order Agents in a TimeVarying Flowfield," IEEE Transactions on Automatic Control, accepted for publication, 2021.

[30] Zhang, Y. and Tian, Y.-P., "Consentability and Protecol Design of Multi-Agent Systems with Stochastic Switching Topology," Automatica, Vol. 45, No. 5, 2009, pp. 1195-1201.

doi:10.1016/j.automatica.2008.11.005

[31] Wang, L. and Xiao, F., "Finite-Time Consensus Problems for Networks of Dynamics Agents," IEEE Transactions on Automatic Control, Vol. 55, No. 4, 2010, pp. 950-955.

doi:10.1109/TAC.2010.2041610

[32] Ren, W., "Consensus Strategies for Cooperative Control of Formations," IET Control Theory E Applications Vol. 1, No. 2, 2007, pp. 505-512.

doi:10.1049/iet-cta:20050401 